\title{
EFEITO DE SOLUÇÕES ISOTÔNICAS NO EQUILÍBRIO ACIDOBÁSICO EM POTROS SADIOS
}

\author{
SANTOS, Alice Correa ${ }^{1}$; \\ SANTOS, Rodrigo Stauffert ${ }^{2}$; \\ CURCIO, Bruna da Rosa ${ }^{3}$; \\ HONSY, Simone Ramalho ${ }^{4}$; \\ DEL PINO, Francisco Augusto Burkert ${ }^{5}$; \\ NOGUEIRA, Carlos Eduardo Wayne ${ }^{3}$.
}

Recebido: 26/11/2015

Aceito: 28/09/2016

\footnotetext{
${ }^{1}$ Programa de Pós-Graduação em Veterinária, UFPEL; ${ }^{2}$ Médico Veterinário Autônomo; ${ }^{3}$ Professor, Doutor, Departamento de Clínicas Veterinárias, UFPEL; ${ }^{4}$ Farmacêutica, Empresa Brasileira de Serviços Hospitalares ; Professor, Doutor, Departamento de Bioquímica, UFPEL.
}

\section{RESUMO}

$\mathrm{N}$ a rotina da clínica médica de equinos, as soluções isotônicas são bastante utilizadas. Entretanto, pouco se sabe sobre seu real efeito no equilíbrio acidobásico em equinos jovens. O objetivo deste estudo foi avaliar o efeito das soluções poliônicas: cloreto de sódio $0,9 \%$, solução de Ringer com lactato e Ringer com lactato acrescido de bicarbonato de sódio sobre o equilíbrio acidobásico em potros. $O$ experimento foi realizado com quatro potros de 18-24 meses, que receberam as três soluções intravenosas em diferentes momentos. Foram realizadas cinco coletas para hemogasometria (T0, T20, T60, T100 e $\mathrm{T} 140)$, sendo avaliado o $\mathrm{pH}$, pressão parcial de dióxido de carbono $\left(\mathrm{pCO}_{2}\right)$, pressão parcial de oxigênio $\left(\mathrm{pO}_{2}\right)$, saturação de hemoglobina por oxigênio $\left(\mathrm{sO}_{2}\right)$, bicarbonato de sódio $\left(\mathrm{HCO}_{3}{ }^{-}\right)$e excesso ou déficit de bases no sangue (BE). A administração de Ringer com lactato acrescido com bicarbonato de sódio promoveu o incremento nas concentrações das variáveis $\mathrm{pH}$, $\mathrm{HCO}_{3}{ }^{-}$e $\mathrm{BE}$, enquanto as demais soluções avaliadas não apresentaram diferença. Embora tenha potencial alcalinizante, a solução de Ringer com lactato acrescida de bicarbonato de sódio demonstrou efeito transitório.

Palavras-chave: Fluidoterapia. Hemogasometria. Equinos. 


\section{INTRODUÇÃO}

A manutenção da homeostase corporal é dependente do equilíbrio entre líquidos corporais, eletrólitos e pH (GOMES, 2013). Para isto, o equilíbrio acidobásico é fundamental, já que a desestabilização desse mecanismo pode levar a disfunções em diversos sistemas (RHODES; CUSACK, 2000). Em equinos, dentre as principais enfermidades que cursam com desequilíbrio acidobásico estão desidratação, síndrome cólica, diarreia, taquipneia decorrente de obstruções respiratórias e insuficiência renal (REED et al., 2004).

Uma importante ferramenta de diagnóstico e acompanhamento clínico do estado acidobásico é a hemogasometria, capaz de determinar o pH sanguíneo, pressão parcial de oxigênio $\left(\mathrm{pO}_{2}\right)$ e pressão parcial de dióxido de carbono $\left(\mathrm{pCO}_{2}\right)$. Com base nestas, calcula-se a saturação de hemoglobina por oxigênio $\left(\mathrm{sO}_{2}\right)$, bicarbonato de sódio $\left(\mathrm{HCO}_{3}{ }^{-}\right)$e excesso ou déficit de bases no sangue (BE) (RIESER, 2013). Os resultados obtidos na hemogasometria possuem correspondência com a condição momentânea do organismo, servindo como subsídio para intervenção e correção de eventuais desequilíbrios de maneira correta (SUCUPIRA; ORTOLANI, 2003).

Na prática da clínica de equinos, a administração de bicarbonato de sódio é usual em casos de acidose metabólica, em razão de sua ação alcalinizante (KLINE et al., 2005). Entretanto, o uso desse tampão em animais deve ser feito com cautela, porque o excesso pode trazer danos como alcalose sistêmica iatrogênica, edema cerebral e acidose paradoxal do fluido cérebro-espinhal (LEAL et al., 2007).

Como alternativa ao uso de solução de bicarbonato de sódio, o Ringer com lactato tem função alcalinizante, sendo a única solução poliônica disponível comercialmente no Brasil (LISBOA et al., 2009). Contudo, pouco se sabe se esta solução é capaz de, através da elevação do pH sanguíneo, alterar a condição acidobásica em equinos jovens (COSENZA et al., 2013).

O objetivo do presente estudo foi avaliar o efeito das soluções isotônicas de cloreto de sódio 0,9\%, Ringer com lactato e Ringer com lactato acrescido de bicarbonato de sódio sobre o equilíbrio acidobásico em potros sadios. 


\section{MATERIAL E MÉTODOS}

Foi realizado um estudo experimental prospectivo em parcelas subdivididas, com todos os potros passando por todos os tratamentos. Foram utilizados quatro potros hígidos, machos, sem raça definida, com idades entre 18 e 24 meses, com escore corporal de seis a sete (HENNECKE et al., 1983) e peso corporal entre 180 e $200 \mathrm{~kg}$. Os animais eram de propriedade do Hospital de Clínicas Veterinárias da Universidade Federal de Pelotas (HCV/UFPEL) e foram alojados em piquetes coletivos com pastagem de azevém (Lolium multiflorum), recebendo ração comercial duas vezes ao dia e água ad libitum. Os animais foram submetidos a controle parasitário, e além de se apresentarem saudáveis ao exame físico e ao hemograma, ainda se mostraram negativos na pesquisa de hemoparasitas.

Cada equino participou dos três tratamentos, totalizando 12 observações, com um intervalo de cinco dias entre os tratamentos. Após cada tratamento, os equinos foram alojados em baias individuais durante o período de coleta das amostras sanguíneas, com privação de água e alimento. Todos os tratamentos foram realizados no período da manhã e com início no mesmo horário. Foram utilizadas soluções de Ringer com lactato, cloreto de sódio a 0,9\% e solução de Ringer com lactato acrescida de bicarbonato de sódio 1,34 mEq/mL.

A solução de Ringer com lactato acrescida de bicarbonato de sódio (Ringer com lactato + $\mathrm{HCO}_{3}{ }^{-}$) foi preparada usando uma técnica estéril em laboratório e foi mensurado o $\mathrm{pH}$ de todas as soluções, antes da administração, em pHmetro calibrado (Kasvi $\left.{ }^{\circledR}, \mathrm{K} 39-2014 \mathrm{~B}\right)$. A concentração de bicarbonato de sódio e volume da solução infundida foram preparados com base no experimento de Rivas et al. (1997) que utilizaram uma solução de $11,25 \%$ de bicarbonato de sódio para induzir alcalose metabólica significativa.

As soluções estudadas foram infundidas por via intravenosa com a utilização de cateter intravenoso número $14 \mathrm{G}$ (Solidor ${ }^{\circledR}$ ) em cada um dos equinos, a uma velocidade média de 50 $\mathrm{mL}$ por minuto totalizando $1000 \mathrm{~mL}$ por animal em 20 minutos de administração contínua.

Para a análise gasométrica, amostras de sangue foram coletadas assepticamente e anaerobicamente, através de punção da veia jugular direita, utilizando agulha hipodérmica

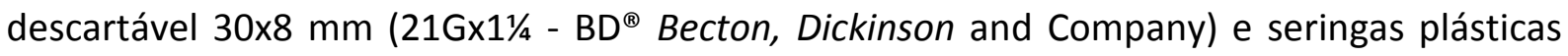
descartáveis de $3 \mathrm{~mL}$ (BD ${ }^{\circledR}$ Becton, Dickinson and Company), com heparina. As amostras 
foram acondicionadas em caixa de isopor com gelo e analisadas em um período máximo de uma hora após a coleta. As coletas sanguíneas foram realizadas antes da infusão da solução (T0), 20 minutos após o término da infusão da solução (T20), e a cada 40 minutos totalizando mais três coletas após o término da infusão da solução (T60, T100 e T140). As coletas foram repetidas nos três tratamentos.

As variáveis analisadas foram o $\mathrm{pH}$ sanguíneo, $\mathrm{pO}_{2}, \mathrm{pCO}_{2}, \mathrm{HCO}_{3}{ }^{-}, \mathrm{sO}_{2}$ e $\mathrm{BE}$. Essas análises foram realizadas em aparelho de gasometria (Omni C; Roche; EUA), com calibração automática diária.

Os dados foram submetidos ao teste de Shapiro Wilk para avaliação da normalidade. As variáveis paramétricas foram testadas por análise de variância e comparação entre as médias pelo teste LSD. As variáveis não paramétricas foram submetidas ao teste de KruskalWallis. A comparação entre as médias foi realizada entre os grupos de tratamento em cada momento e entre os momentos avaliados dentro de cada grupo. Todas as análises foram realizadas com o auxílio do software Statistix $8.0^{\circledR}$ (Analytical Software, Tallahasse, FL, USA). A significância foi atribuída aos valores de $p<0,05$. Todos os valores estão expressos em média \pm desvio padrão (DP).

Todos os procedimentos realizados nos animais foram aprovados pelo Comitê de Ética e Experimentação Animal da Faculdade de Veterinária da Universidade Federal de Pelotas, número 6902.

\section{RESULTADOS E DISCUSSÃO}

Os valores observados nas variáveis hemogasométricas em cada uma das soluções entre os momentos estão descritos nas Tabelas 1, 2 e 3. Destaca-se que as soluções $\mathrm{NaCl}$ 0,9\% e Ringer com lactato não apresentaram diferença em nenhuma das variáveis entre os momentos após a administração (T20, T60, T100 e T140). 
Tabela 1 - Médias das variáveis $\mathrm{pH}, \mathrm{pO}_{2}(\mathrm{mmHg}), \mathrm{pCO}_{2}(\mathrm{mmHg}), \mathrm{HCO}_{3}{ }^{-}(\mathrm{mEq} / \mathrm{L}), \mathrm{sO}_{2}(\%)$ e $\mathrm{BE}(\mathrm{mEq} / \mathrm{L}) \mathrm{nos}$ momentos após a administração de $\mathrm{NaCl} 0,9 \%$.

\begin{tabular}{llllll}
\hline \multicolumn{5}{c}{$\mathrm{NaCl} 0,9 \%$} \\
& $\mathrm{T0}$ & $\mathrm{T} 20$ & $\mathrm{T60}$ & $\mathrm{T} 100$ & $\mathrm{~T} 140$ \\
\hline $\mathrm{pH}$ & $7,406 \pm 0,017 \mathrm{a}$ & $7,399 \pm 0,02 \mathrm{a}$ & $7,409 \pm 0,02 \mathrm{a}$ & $7,400 \pm 0,001 \mathrm{a}$ & $7,395 \pm 0,02 \mathrm{a}$ \\
$\mathrm{pO}_{2}$ & $39,08 \pm 3,32 \mathrm{a}$ & $39,68 \pm 3,22 \mathrm{a}$ & $42,93 \pm 5,77 \mathrm{a}$ & $42,6 \pm 3,16 \mathrm{a}$ & $41,93 \pm 3,32 \mathrm{a}$ \\
$\mathrm{pCO}_{2}$ & $49,48 \pm 1,36 \mathrm{a}$ & $50,05 \pm 2,16 \mathrm{a}$ & $47,13 \pm 1,82 \mathrm{a}$ & $47,15 \pm 1,91 \mathrm{a}$ & $48,75 \pm 2,93 \mathrm{a}$ \\
$\mathrm{HCO}_{3}^{-}$ & $30,33 \pm 1,02 \mathrm{a}$ & $30,15 \pm 0,81 \mathrm{a}$ & $29,15 \pm 0,82 \mathrm{a}$ & $28,58 \pm 1,18 \mathrm{a}$ & $29,18 \pm 1,22 \mathrm{a}$ \\
$\mathrm{SO}_{2}$ & $74,48 \pm 3,86 \mathrm{a}$ & $74,80 \pm 5,34 \mathrm{a}$ & $78,73 \pm 6,26 \mathrm{a}$ & $78,35 \pm 4,00 \mathrm{a}$ & $77,00 \pm 4,43 a$ \\
$\mathrm{BE}$ & $4,63 \pm 1,16 \mathrm{a}$ & $4,60 \pm 0,82 \mathrm{a}$ & $3,85 \pm 0,63 \mathrm{a}$ & $3,20 \pm 1,02 \mathrm{a}$ & $3,63 \pm 1,21 \mathrm{a}$
\end{tabular}

T0: antes da infusão da solução; T20: 20 minutos após o término da infusão da solução; T60: 60 minutos após o término da infusão da solução; T100: 100 minutos após o término da infusão da solução; T140: 140 minutos após o término da infusão da solução. Letras minúsculas $(a, b)$ representam diferença significativa $(p<0,05)$ entre os momentos.

Tabela 2 - Média e desvio padrão das variáveis $\mathrm{pH}, \mathrm{pO}_{2}(\mathrm{mmHg}), \mathrm{pCO}_{2}(\mathrm{mmHg}), \mathrm{HCO}_{3}{ }^{-}(\mathrm{mEq} / \mathrm{L}), \mathrm{sO}_{2}(\%)$ e BE (mEq/L) nos momentos após a administração de Ringer com lactato.

$$
\text { Ringer com lactato }
$$

\begin{tabular}{llllll} 
& T0 & T20 & T60 & T100 & T140 \\
\hline $\mathrm{pH}$ & $7,403 \pm 0,016 \mathrm{a}$ & $7,404 \pm 0,02 \mathrm{a}$ & $7,399 \pm 0,01 \mathrm{a}$ & $7,401 \pm 0,01 \mathrm{a}$ & $7,402 \pm 0,011 \mathrm{a}$ \\
$\mathrm{pO}_{2}$ & $40,15 \pm 2,36 \mathrm{a}$ & $42,63 \pm 4,92 \mathrm{a}$ & $38,83 \pm 3,20 \mathrm{a}$ & $39,05 \pm 5,99 \mathrm{a}$ & $44,18 \pm 2,62 \mathrm{a}$ \\
$\mathrm{pCO}_{2}$ & $48,50 \pm 1,99 \mathrm{a}$ & $48,58 \pm 2,60 \mathrm{a}$ & $48,88 \pm 1,63 \mathrm{a}$ & $49,63 \pm 2,00 \mathrm{a}$ & $47,53 \pm 2,40 \mathrm{a}$ \\
$\mathrm{HCO}_{3}{ }^{-}$ & $29,55 \pm 1,24 \mathrm{a}$ & $29,65 \pm 0,78 \mathrm{a}$ & $29,48 \pm 0,56 \mathrm{a}$ & $30,08 \pm 0,94 \mathrm{a}$ & $28,93 \pm 1,06 \mathrm{a}$ \\
$\mathrm{sO}_{2}$ & $75,75 \pm 3,48 \mathrm{a}$ & $78,35 \pm 5,64 \mathrm{a}$ & $73,53 \pm 4,89 \mathrm{a}$ & $73,33 \pm 8,38 \mathrm{a}$ & $80,25 \pm 3,39 \mathrm{a}$ \\
$\mathrm{BE}$ & $4,00 \pm 1,04 \mathrm{a}$ & $4,20 \pm 0,78 \mathrm{a}$ & $4,03 \pm 0,41 \mathrm{a}$ & $4,28 \pm 0,65 \mathrm{a}$ & $3,40 \pm 0,80 \mathrm{a}$ \\
\hline
\end{tabular}

T0: antes da infusão da solução; T20: 20 minutos após o término da infusão da solução; T60: 60 minutos após o término da infusão da solução; T100: 100 minutos após o término da infusão da solução; T140: 140 minutos após o término da infusão da solução. Letras minúsculas $(a, b)$ representam diferença significativa $(p<0,05)$ entre os momentos. 
Tabela 3 - Média e desvio padrão das variáveis $\mathrm{pH}, \mathrm{pO}_{2}(\mathrm{mmHg}), \mathrm{pCO}_{2}(\mathrm{mmHg}), \mathrm{HCO}_{3}{ }^{-}(\mathrm{mEq} / \mathrm{L}), \mathrm{sO}_{2}(\%)$ e $\mathrm{BE}$ (mEq/L) nos momentos após a administração de Ringer com lactato com bicarbonato de sódio.

Ringer com lactato com bicarbonato de sódio

\begin{tabular}{llllll} 
& T0 & T20 & T60 & T100 & T140 \\
\hline $\mathrm{pH}$ & $7,391 \pm 0,02 \mathrm{~b}$ & $7,525 \pm 0,01 \mathrm{a}$ & $7,451 \pm 0,02 \mathrm{ab}$ & $7,410 \pm 0,02 \mathrm{ab}$ & $7,405 \pm 0,01 \mathrm{ab}$ \\
$\mathrm{pO}_{2}$ & $37,55 \pm 1,12 \mathrm{a}$ & $39,53 \pm 2,88 \mathrm{a}$ & $37,10 \pm 0,74 \mathrm{a}$ & $39,05 \pm 1,23 \mathrm{a}$ & $40,78 \pm 3,29 \mathrm{a}$ \\
$\mathrm{pCO}_{2}$ & $51,70 \pm 1,78 \mathrm{a}$ & $54,38 \pm 3,04 \mathrm{a}$ & $55,25 \pm 3,66 \mathrm{a}$ & $52,75 \pm 3,02 \mathrm{a}$ & $53,50 \pm 2,34 \mathrm{a}$ \\
$\mathrm{HCO}_{3}^{-}$ & $31,13 \pm 0,57 \mathrm{~b}$ & $40,43 \pm 0,74 \mathrm{a}$ & $35,55 \pm 0,85 \mathrm{ab}$ & $32,75 \pm 0,93 \mathrm{ab}$ & $32,63 \pm 1,50 \mathrm{ab}$ \\
$\mathrm{sO}_{2}$ & $71,70 \pm 1,93 \mathrm{~b}$ & $77,88 \pm 3,67 \mathrm{a}$ & $72,28 \pm 1,80 \mathrm{~b}$ & $75,23 \pm 2,63 \mathrm{ab}$ & $76,95 \pm 3,84 \mathrm{a}$ \\
$\mathrm{BE}$ & $5,13 \pm 0,50 \mathrm{~b}$ & $10,35 \pm 0,64 \mathrm{a}$ & $7,68 \pm 1,35 \mathrm{ab}$ & $6,78 \pm 1,83 \mathrm{ab}$ & $6,80 \pm 0,79 \mathrm{ab}$
\end{tabular}

T0: antes da infusão da solução; T20: 20 minutos após o término da infusão da solução; T60: 60 minutos após o término da infusão da solução; T100: 100 minutos após o término da infusão da solução; T140: 140 minutos após o término da infusão da solução. Letras minúsculas $(a, b)$ representam diferença significativa $(p<0,05)$ entre os momentos.

No grupo Ringer com lactato $+\mathrm{HCO}_{3}{ }^{-}$, vinte minutos após a administração (T20), a mensuração do $\mathrm{HCO}_{3}{ }^{-}$foi superior aos demais momentos, em função da alcalinização imediata que esse tampão provocou, corroborando a utilização universal dessa solução para a correção de acidose metabólica (ÉVORA et al., 1999). O aumento do BE após administração da solução (T20) e a manutenção desses valores elevados nos momentos seguintes evidencia a ação alcalinizante dessa solução, já que o excesso de bases é decorrente do incremento alcalino no sangue (FREITAS et al., 2010).

Nas Figuras 1 e 2 observam-se as variações na mensuração de $\mathrm{HCO}_{3}{ }^{-}$e $\mathrm{BE}$ sanguíneos com a infusão das diferentes soluções. Ambas variáveis demonstraram as maiores variações durante a administração nas diferentes soluções. 


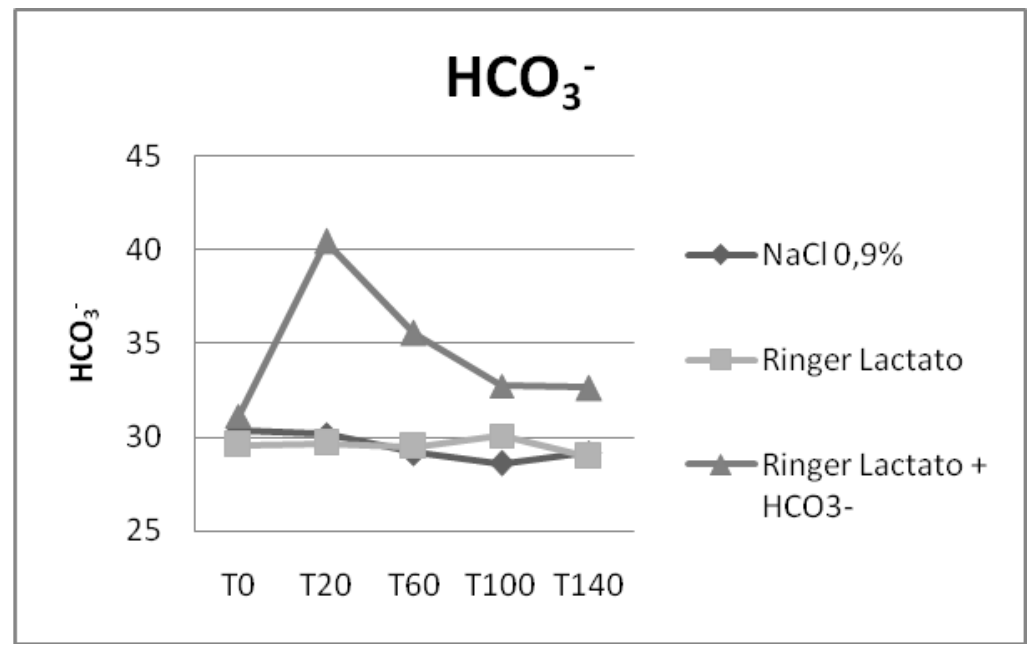

Figura 1 - Variação do $\mathrm{HCO}_{3}{ }^{-}$sanguíneo frente às soluções de $\mathrm{NaCl} 0,9 \%$, Ringer com lactato e Ringer com lactato $+\mathrm{HCO}_{3}{ }^{-}$na administração (T0), imediatamente após o término da infusão (T20) e aos 60, 100 e 140 minutos pós infusão.

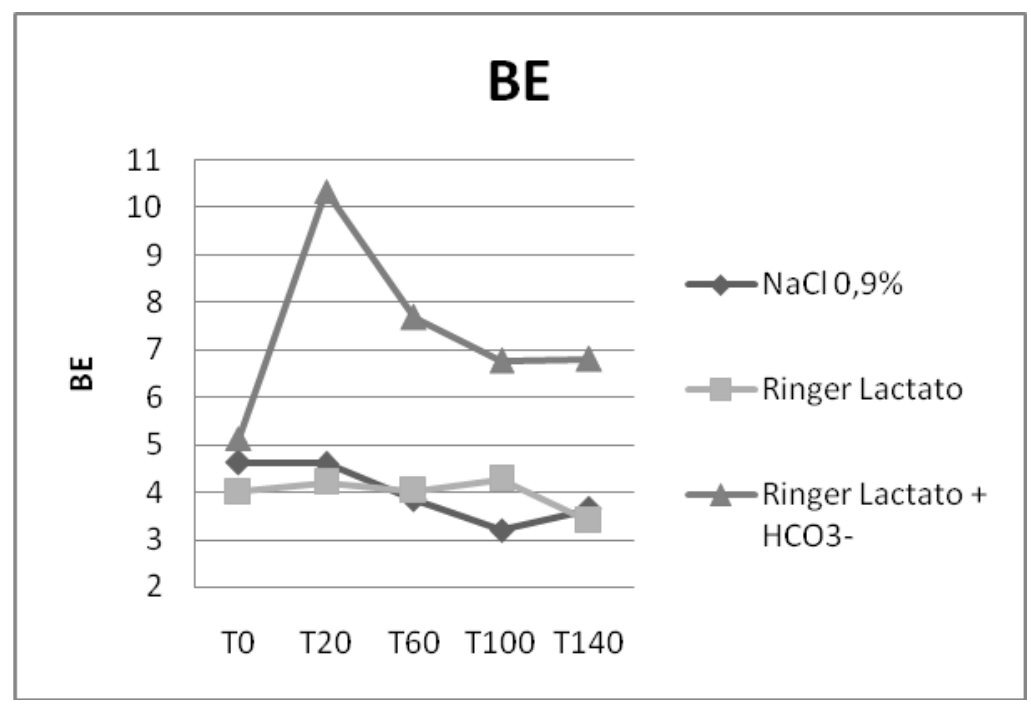

Figura 2 - Variação do BE sanguíneo frente às soluções de $\mathrm{NaCl}$ 0,9\%, Ringer com lactato e Ringer com lactato + $\mathrm{HCO}_{3}{ }^{-}$na administração (TO), imediatamente após o término da infusão (T20) e aos 60, 100 e 140 minutos pós infusão.

Os gráficos das Figuras 1 e 2 demonstram curvas semelhantes, contudo é possível observar que na administração da solução de Ringer com lactato $+\mathrm{HCO}_{3}{ }^{-}$, a queda nos níveis de $\mathrm{HCO}_{3}{ }^{-}$ ocorre rápida e constantemente após a administração (T20), voltando próximo ao basal a 
partir de T100. Já os valores do BE sofrem queda e se mantém estabilizados por um período maior.

Esse incremento de $\mathrm{HCO}_{3}{ }^{-}$é evidente, por coincidir com o momento da administração de bicarbonato de sódio. $\mathrm{O}$ mesmo foi observado com o $\mathrm{BE}$, que aumenta em decorrência de alcalose (FREITAS et al., 2010), nesse caso provocada pela infusão da solução de $\mathrm{HCO}_{3}{ }^{-}$. A elevação dos níveis de $\mathrm{HCO}_{3}{ }^{-}$no sangue venoso ocorreu na administração da solução de Ringer com lactato $+\mathrm{HCO}_{3}{ }^{-}$, entretanto esse aumento foi transitório, reduzindo aos níveis basais por volta de 100 minutos (T100).

Os valores da $\mathrm{sO}_{2}$ e $\mathrm{pO}_{2}$ mensurados no presente estudo não foram utilizados para avaliação, pois segundo Gomes (2013) são melhor avaliados em sangue arterial por serem menos sensíveis a alterações metabólicas. Apesar da $\mathrm{sO}_{2}$ e $\mathrm{pO}_{2}$ serem parâmetros mais confiáveis no sangue arterial, cabe ressaltar que a coleta do sangue venoso é de fácil execução na rotina clínica, e produz outros resultados fidedignos acerca do equilíbrio acidobásico (DAY, 2002). A p $\mathrm{pCO}_{2}$ não apresentou diferença em nenhuma das soluções estudadas.

A Figura 3 apresenta a discrepância na variação do pH após a administração da solução de Ringer com lactato $+\mathrm{HCO}_{3}{ }^{-}$em comparação com as demais soluções.

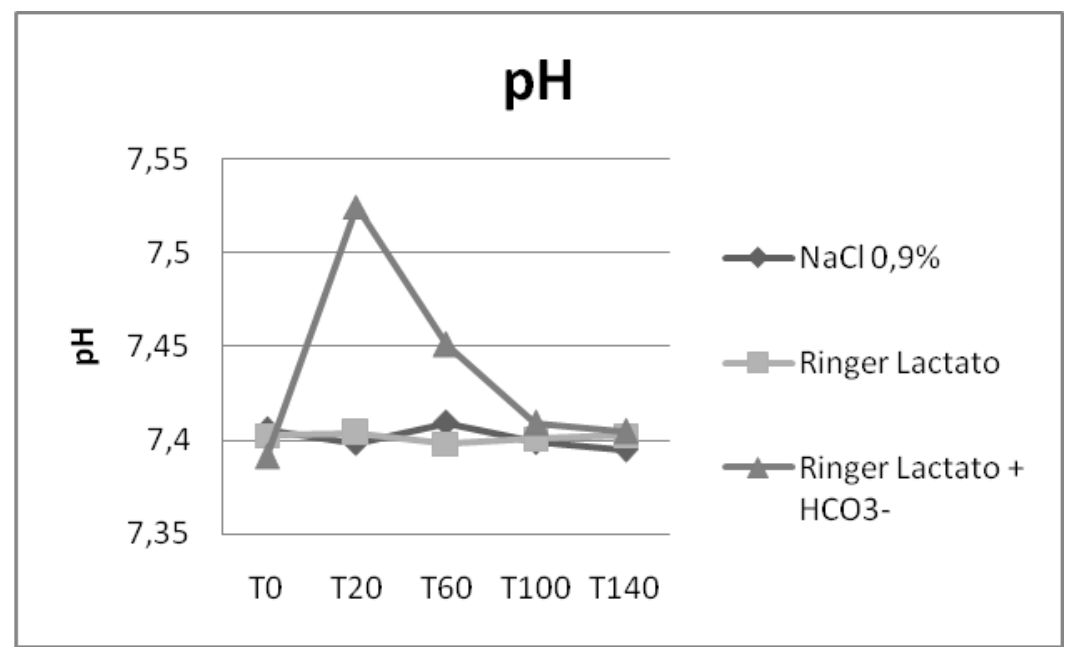

Figura 3 - Variação do pH no sanguíneo frente as soluções de $\mathrm{NaCl} 0,9 \%$, Ringer com lactato e Ringer com lactato $+\mathrm{HCO}_{3}^{-}$na administração (T0), imediatamente após o término da infusão (T20) e aos 60, 100 e 140 minutos pós infusão. 
$\mathrm{O}$ pH demonstrou elevação no grupo Ringer com lactato $+\mathrm{HCO}_{3}{ }^{-}$, a partir do $\mathrm{T} 20(\mathrm{p}=0,024)$ até T60 $(p=0,026)$. Essa elevação decorre da atividade alcalinizante do bicarbonato, pela ação no equilíbrio acidobásico, o que está diretamente relacionado à capacidade de tamponamento do sangue (FREITAS et al., 2010).

Nas soluções de Ringer com lactato, o mecanismo que alcaliniza o pH sanguíneo decorre da atividade precursora de bases do lactato, sendo que íons bicarbonato $\left(\mathrm{HCO}_{3}{ }^{-}\right)$são, direta ou indiretamente, gerados durante esse processo de metabolização (CONSTABLE, 2003) através da via neoglicogênica e de reações oxidativas, elevando o pH (KASARI, 1999).

Entretanto, neste trabalho a solução de Ringer com lactato não produziu mudança significativa no pH sanguíneo, que segundo Lisboa et al. (2009) pode estar relacionado com a baixa concentração de lactato na solução $(28 \mathrm{mEq} / \mathrm{L})$. Estudos em bezerros demonstraram que soluções com maior concentração de lactato $(84 \mathrm{mEq} / \mathrm{L})$ tendem a aumentar a reserva alcalina (JUNQUEIRA et al., 2015). É provável que não tenham ocorrido mudanças no pH sanguíneo após a administração de solução de Ringer com lactato devido à baixa concentração na solução utilizada, que é padrão para as soluções disponíveis comercialmente no Brasil.

Embora existam poucos estudos acerca do metabolismo do lactato sobre o equilíbrio acidobásico em potros, sugere-se que seja semelhante ao que ocorre em adultos. Conforme observado em experimento com equinos adultos mestiços, a solução de Ringer com lactato não aumentou a reserva alcalina, porque a pouca quantidade de lactato presente foi rapidamente metabolizada. Como conseqüência, acabam sendo convertidos valores insignificantes de lactato em $\mathrm{HCO}_{3}^{-}$, que não demonstram diferenças significativas no equilíbrio acidobásico (COSENZA et al., 2013).

A síndrome cólica é uma das principais enfermidades observadas na clínica de equinos, que geralmente cursa com desequilíbrios eletrolíticos de sódio, potássio e magnésio (REED et al., 2004). Além destes distúrbios, Castro e González (2015) demonstraram em animais com síndrome cólica a elevação do $\mathrm{pH}, \mathrm{pCO}_{2}, \mathrm{HCO}_{3}{ }^{-}$e $\mathrm{BE}$, especialmente quando existiam lesões de intestino delgado, indicando condição de alcalose metabólica. Entretanto, de forma errônea, na prática da clínica de equinos muitas vezes são administradas soluções 
alcalinizantes com o objetivo de corrigir uma possível acidose, podendo agravar ainda mais esta situação de alcalose metabólica pré-estabelecida.

Nos equinos em exercício, foi demonstrado que a utilização de soluções acrescidas de $\mathrm{HCO}_{3}{ }^{-}$ podem causar desequilíbrios eletrolíticos, como hipocalcemia, hipocalemia e hipernatremia (KLINE, 1995). Este dado demonstra o quanto é importante o conhecimento do equilíbrio acidobásico do paciente para a escolha da solução correta, evitando assim, o desequilíbrio acido básico iatrogênico.

Cabe reafirmar que o $\mathrm{HCO}_{3}{ }^{-}$até o presente momento é a única solução capaz de alcalinizar o pH em potros, mesmo com efeito transitório. Entretanto, a utilização de uma solução alcalinizante sem o conhecimento do estado acidobásico do paciente pode ser danosa, evidenciando a importância da hemogasometria para o acompanhamento adequado. Sabese que a hemogasometria ainda é um recurso vinculado aos grandes centros de referência, contudo atualmente existem aparelhos portáteis que podem fornecer estes parâmetros também a campo, e havendo a disponibilidade devem ser utilizados.

\section{CONCLUSÃO}

Nos potros de 18 a 24 meses a solução de Ringer com lactato não apresentou efeito alcalinizante. Entretanto, a solução de Ringer com lactato acrescida de bicarbonato de sódio demonstrou potencial alcalinizante, porém com efeito transitório.

\section{THE EFFECT OF ISOTONIC SOLUTIONS ON ACID BASIC BALANCE IN HEALTHY FOALS}

\section{ABSTRACT}

$\mathrm{n}$ the equine medical routine, isotonic solutions are widely used. However, the effect of these solutions on acid-base balance in young horses remains unknown. The aim of this study is evaluate the effect of these polionic solutions: $\mathrm{NaCl} 0.9 \%$, Ringer's lactate and Ringer's lactate plus sodium bicarbonate (Ringer's lactate $+\mathrm{HCO}_{3}{ }^{-}$) on acid-base status in foals. The experiment was conducted with four foals at 18-24 months of age, which received tree intravenous solutions at different times. Venous blood was collected for blood gas analysis in five moments, before and after-infusion (T0, T20, T60, T100 e T140). The pH, 
partial pressure of carbon dioxide $\left(\mathrm{pCO}_{2}\right)$, partial pressure of oxygen $\left(\mathrm{pO}_{2}\right)$, oxygen saturation $\left(\mathrm{sO}_{2}\right)$, sodium bicarbonate $\left(\mathrm{HCO}_{3}{ }^{-}\right)$and base excess $(\mathrm{BE})$ were evaluated. The infusion of Ringer's lactate $+\mathrm{HCO}_{3}{ }^{-}$increased the concentration of $\mathrm{pH}, \mathrm{pO}_{2}, \mathrm{pCO}_{2}, \mathrm{HCO}_{3}{ }^{-}$and $\mathrm{BE}$. The infusion of $\mathrm{NaCl} 0.9 \%$ and Ringer lactate's solution did not result in changes on blood gas analysis. However, the infusion of Ringer lactate $+\mathrm{HCO}_{3}{ }^{-}$showed transient alkalizing effects in foals.

Keywords: Fluid. Hemogasometry. Horses.

\section{EFECTO DE SOLUCIONES ISOTONICAS EN EL EQUILIBRIO ÁCIDO-BASE EN POTROS SALUDABLES}

\section{RESUMEN}

$\square$ n la clínica médica equina, son ampliamente utilizados soluciones isotónicas. Sin embargo, se sabe poco sobre su efecto sobre el equilibrio ácido-base en caballos jóvenes. El objetivo de este estudio fue evaluar el efecto de las soluciones poli-iónicas como cloruro de sodio al $0,9 \%$, Ringer con lactato e Ringer con lactato con el agregado de bicarbonato de sodio sobre el equilibrio ácido-base en potros. El experimento fue realizado con cuatro potros de 18-24 meses de edad, que recibieron las tres soluciones intravenosas en diferentes momentos. Fue obtenida la sangre venosa para el análisis de gases en cinco tiempos antes y después de la infusión (T0, T20, T60, T100 y T140), siendo evaluado el pH, $\mathrm{pCO}_{2}, \mathrm{pO}_{2}, \mathrm{SO}_{2}, \mathrm{HCO}_{3}$ y $\mathrm{BE}$. La administración de Ringer con lactato $+\mathrm{HCO}_{3}^{-}$promovió el aumento de las concentraciones de las variables $\mathrm{pH}, \mathrm{HCO}_{3}{ }^{-}$y $\mathrm{BE}$. Las soluciones $\mathrm{NaCl} 0,9 \%$ y Ringer con lactato no cambiaran expresivamente el equilibrio ácido-base de los potros. $\mathrm{A}$ pesar de tener potencial alcalinizante, la solución Ringer con lactato con el agregado de bicarbonato de sodio presentó potencial alcalinizante transitorio.

Palabras clave: Fluido. Hemogasometria. Caballos.

\section{REFERÊNCIAS}

CASTRO, T. F.; GONZÁLEZ, F. Blood gas analysis in Mangalarga Marchador horses with colic. Revista MVZ Córdoba, v. 20, n. 1, p. 4447-4454, 2015.

CONSTABLE, P. D. Fluid and electrolyte therapy in ruminants. Veterinary Clinics of North America: Food Animal Practice, v. 19, n. 3, p. 557-597, 2003. 
COSENZA, M.; PEREIRA, P. F. V.; FERNANDES, L. I.; et al. Efeito da solução de Ringer com lactato sobre os equilíbrios hidroeletrolítico e acidobase de equinos, ovelhas e bezerros sadios. Ciência Rural, v. 43, n. 12, p. 2247-2253, 2013.

DAY, T. K. Blood gas analysis. Veterinary Clinics of North America: Small Animal Practice, v. 32, n. 5, p. 1031-1048, 2002.

ÉVORA, P. R. B.; REIS, C. L.; FEREZ, M. A.; et al. Distúrbios do equilíbrio hidroeletrolítico e do equilíbrio acidobásico - Uma revisão prática. Medicina, v. 32, n. 4, p. 451-469, 1999.

FREITAS, M. D.; FERREIRA, M. G.; FERREIRA, P. M.; et al. Equilíbrio eletrolítico e ácido-base em bovinos. Ciência Rural, v. 40, n. 12, p. 2608-2615, 2010.

GOMES, A. V. Análise hemogasométrica do sangue venoso equino pré e pós-exercício. Porto Alegre: UFRGS, 2013. 40p. Dissertação (Mestrado em Medicina Animal), Universidade Federal do Rio Grande do Sul, 2013.

HENNECKE, D. R.; POTTER, G. D.; KREIDER, J. L.; et al. Relationship between condition score, physical measurements and body fat percentage in mares. Equine Veterinary Journal, v. 15, n. 4, p. 371-371, 1983.

JUNQUEIRA, J. R. C.; BALARIN, M. R. S; FLAIBAN, K. K. M. C.; et al. Efeito alcalinizante de soluções eletrolíticas intravenosas com concentrações elevadas de lactato de sódio infundidas em bezerros sadios. Arquivo Brasileiro de Medicina Veterinária e Zootecnia, $v$. 67, n. 1, p. 15-24, 2015.

KASARI, T. R. Metabolic acidosis in calves. Veterinary Clinics of North America: Food Animal Practice, v. 15, n. 3, p. 473-486, 1999.

KLINE, K.; FREY, L. P.; FOREMAN, J. H.; et al. Effects of intravenous sodium bicarbonate and sodium acetate on equine acid-base status. Journal of Equine Veterinary Science, v. 25, n. 8, p. 349-354, 2005.

LEAL, M. L. R.; MARUTA, C. A.; ORTOLANI, E. L. Uso de bicarbonato e lactato-L para correção da acidose metabólica sistêmica em bovinos com acidose láctica ruminal aguda. Arquivo Brasileiro de Medicina Veterinária e Zootecnia, v. 59, n. 4, p. 971-976, 2007.

REED, S. M.; BAYLY, M. W.; SELLON, D. C. Equine Internal Medicine. 2. ed. Philadelphia: W. B. Saunders, 2004. 1659p.

RHODES, A.; CUSACK, R. J. Arterial blood gas analysis and lactate. Current Opinion in Critical Care, v. 6, n. 3, p. 227-231, 2000.

RIESER, T. M. Arterial and venous blood gas analyses. Topics in Companion Animal Medicine, v. 28, p. 86-90, 2013. 
RIVAS, L. J.; HINCHCLIFF, K. W.; KOHN, C. W.; et al. Effect of sodium bicarbonate administration on blood constituents of horses. American Journal of Veterinary Research, v. 58, n. 6, p. 658-663, 1997.

SUCUPIRA, M. C. A.; ORTOLANI, E. L. Uso de sangue arterial e venoso no exame do equilíbrio ácido-básico de novilhos normais ou com acidose metabólica. Ciência Rural, v. 33, n. 5, p. 863-868, 2003. 